\title{
Severe Acute Necrotizing Pancreatitis Associated with Lipoprotein Lipase Deficiency in Childhood
}

\author{
By L.A. van Walraven, J.B.C. de Klerk, and R.R. Postema \\ Rotterdam, The Netherlands
}

An 11-year-old girl with lipoprotein lipase deficiency experienced recurring episodes of abdominal pain. She initially underwent appendectomy for suspected appendicitis; however, the appendix was normal. Pancreatitis was subsequently identified as the cause of her pain.
J Pediatr Surg 38:1407-1408. (C) 2003 Elsevier Inc. All rights reserved.

INDEX WORDS: Lipoprotein lipase deficiency, acute abdomen, necrotizing pancreatitis.
T HE INCIDENCE of acute pancreatitis in childhood is unknown, but it is suspected to be more common than currently recognized. Diagnosis remains difficult, especially in infancy, because of the nonspecific clinical features and the small window period to establish the correct diagnosis. ${ }^{1-3}$

\section{CASE REPORT}

An 11-year-old girl, known in our center with lipoprotein lipase deficiency (autosomal recessive disorder with an incidence of 1/1.000.000 newborns), was hospitalized elsewhere with severe abdominal pain. The diagnosis of lipoprotein lipase (LPL) was not known. An emergency laparotomy was undertaken during the first admission. She was thought to have peritonitis based on appendicitis, and classic open appendectomy was performed. After 2 days, the abdominal pain disappeared, and the appendicitis was believed to be the source of her symptoms. She left the hospital without complains. The later microscopic findings did not confirmed the diagnosis appendicitis (appendix sana).

The second attack of abdominal pain was 3 months later, and during this hospitalization, she was transferred after 4 days to our center because of clinical decline. During both hospitalizations, the surgical team excluded the diagnosis of acute pancreatitis based on a normal serum amylase concentration (100 IU/L).

We found a very ill girl with severe abdominal and back pain and ileus with persisting vomiting. Temperature was $38.8^{\circ} \mathrm{C}$, and the serum amylase estimation was $379 \mathrm{U} / \mathrm{L}$. The other findings were $\mathrm{C}$ reactive protein (CRP), $36 \mathrm{mg} / \mathrm{L}$; white blood cell count (WBC), 17 10 $\mathrm{E} 9 / \mathrm{L}$; triglyceride level, $33 \mathrm{mmol} / \mathrm{L}$; and lipase level, 5,060 U/L. The patient was not on her diet for LPL so there was a strong suspicion of pancreatitis in this case.

The computed tomography (CT) scan showed an severe pancreatitis (Balthazar E) with 10\% necrosis of the corpus (Computed Topography Severity Index 6) (Fig 1). An intensive therapy was performed on our intensive care unit. The fluid, electrolyte, and mineral balance was restored and maintained, and carention, total parenteral nutrition, and pain relief were given by infusion.

Early antibiotics were started (imipenem-cilastatin) because of a temperature of $39.1^{\circ} \mathrm{C}$, WBC of $1710^{\mathrm{E}} 9 / 1$, and CRP of $211 \mathrm{mg} / \mathrm{L}$. Administration of heparin was not necessary because the plasma triglyceride concentration fell and normalized in 2 days. The second CT scan showed a more severe image with peripancreatic fluid collections but there was clinical improvement, so intensive medical treatment was continued (Fig 2). After 4 weeks of treatment she left the hospital with her diet for LPL without any complains and with normal endocrine and exocrine pancreas functions.

\section{DISCUSSION}

Acute pancreatitis is seen commonly in adult surgical practice. In contrast, acute pancreatitis in children is rare and diagnosis often is delayed. In this case, the massive elevation of plasma triglycerides, which often is clinically silent, resulted in 2 severe attacks of pancreatitis. In our hospital, the triglyceride concentration already dropped and, as a result, the amylase concentration rose (379 U/L). At admission in the other hospital the amylase concentration was normal (100 U/L). The treatment for lipoprotein lipase deficiency is aimed at making the diet low enough in long chain fatty acids to keep the patient asymtomatic and free of recurrent bouts of pain. The diagnosis of pancreatitis was missed because of a diagnostic problem that arose when only the serum amylase concentration was considered in this patient with severe abdominal pain.

The value of serum amylase is reduced by high serum triglycerides, so pancreatitis may occur with a normal amylase concentration in patients with hypertriglyceridaemia. ${ }^{4,5}$ A great variety of etiologies of acute pancreatitis are evident in children; idiopathic (22\%), trauma $(20 \%)$, infection $(15 \%)$, biliary tract disease $(14 \%)$, drugs $(13 \%)$, congenital anomalies $(5 \%)$, hereditary, miscellaneous, and, as in this case, metabolic disorders. ${ }^{5}$

From the Department of General Surgery, Academic Hospital Dijkzigt and the Departments of Metabolic Disorders and Paediatric Surgery, Academic Children's Hospital-Sophia, Rotterdam, The Netherlands.

Address reprint requests to Laurens A. van Walraven, $M D$, Department of Surgery, Academic Hospital Dijkzigt, Dr Molenwaterplein 40, 3015 GD, Rotterdam, The Netherlands.

(C) 2003 Elsevier Inc. All rights reserved.

0022-3468/03/3809-0029\$30.00/0

doi:10.1016/S0022-3468(03)00409-3 


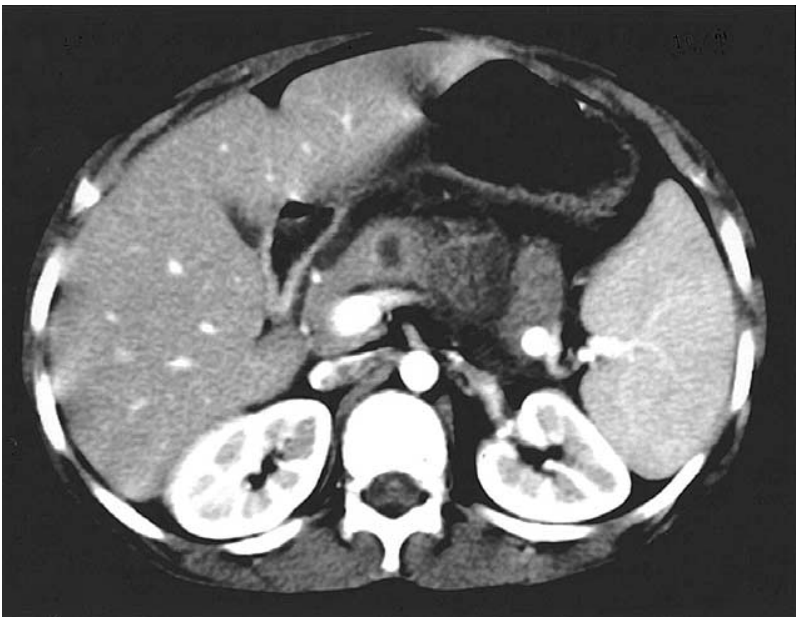

Fig 1. CT scan at admission. Severe acute necrotizing pancreatitis, Balthazar E, with visible necrosis in the corpus of the pancreas.

The clinical features are not specific, but sudden onset of severe abdominal pain in the epigastric upper quadrant may be seen in acute pancreatitis with radiation to the back or left shoulder.

In children with acute pancreatitis, initially, the serum amylase levels are normal in $10 \%$ to $15 \%$ of the patients. Serum lipase is more specific than amylase for acute inflammatory pancreatic disease and should be determined when pancreatitis is suspected, and the amylase

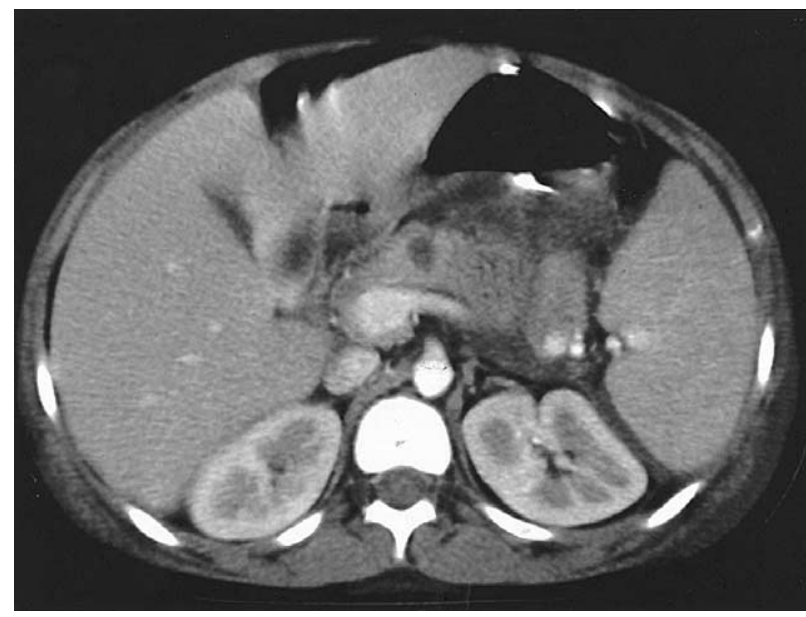

Fig 2. CT scan after 1 week. More peripancreatic collections are visible.

level is normal. Furthermore, these enzyme assays are not useful in infancy because of the physiologic defiency of both enzymes. ${ }^{2,3}$ Ultrasound scan and CT scan are useful in looking for edema, necrosis, abscess, or pseudocyst of the pancreas.

The clinical awareness still is the most important factor leading to correct diagnosis, and hopefully future novel diagnostic markers (serum trypsinogen 2 and alpha-1-antitrypsin). will be commercially available soon.

\section{REFERENCES}

1. Haddock G, Youngson GG, Mackinlay GA, et al: Acute pancreatitis in children: A 15-year review. J Pediatr Surg 6:719-722, 1994

2. Weizman ZVI: Acute pancreatitis in childhood: Research of pathogenesis and clinical implications. Can J Gastroenterol 11:249253, 1997

3. Meyha DI: Acute and chronic pancreatitis in childhood. Indian J Pediatr 66:S81-S86, 1999
4. Ohmoto K, Neishi Y, Miyake I, et al: Severe acute pancreatitis associated with hyperlipidemia: Report of two cases and review of the literature in Japan. Hepatogastroenerol 46:2986-2990, 1999

5. Sharma P, Lim S, James D, et al: Pancreatitis may occur with a normal amylase concentration in hypertriglycerideamia. BMJ 313: 1265,1996 